\title{
Route Prediction on Tracking Data to Location-Based Services
}

\author{
Attila István Petróczi and Csaba Gáspár-Papanek \\ Budapest University of Technology and Economics, \\ Department of Telecommunications and Media Informatics, \\ Magyar Tudósok Krt. 2, 1117 Budapest, Hungary \\ \{petroczi, gaspar\}@tmit.bme.hu \\ http://www.tmit.bme.hu/home!eng
}

\begin{abstract}
Wireless networks have become so widespread, it is beneficial to determine the ability of cellular networks for localization. This property enables the development of location-based services, providing useful information. These services can be improved by route prediction under the condition of using simple algorithms, because of the limited capabilities of mobile stations. This study gives alternative solutions for this problem of route prediction based on a specific graph model. Our models provide the opportunity to reach our destinations with less effort.

Keywords: mobility modeling, route prediction, Markov model, pattern matching model, radio frequency identification (RFID), location-based services (LBS).
\end{abstract}

\section{Introduction}

In our rushing world the modern man demands communication and information channels, that is why they enjoy the benefits of using mobile phones and location systems. The opportunity to use the location-based services (LBS), which are information services accessible with mobile devices through the PLMN (public land mobile network) and utilizing the ability to make use of the location of the mobile device, is given by these equipments. It does not depend on the device used. These services are based on localisation, they use GPS coordinates or information of GSM cells. It is very useful to know where the nearest preferred restaurant or petrol station is, but it can be even more useful to know which one we can reach with the least effort. It means that the system offers us the place which will be the closest to our route in the following few minutes. It is significant in these applications that the algorithm is simple because the mobile devices have small capacity of memory, storage and computing. Our aim is to give such solutions that are very simple and easy to implement, to predict mobility.

\section{Related Works}

There were many research projects about route prediction in GSM and ATM systems in the past and nowadays there are some researches dealing with the 
latest technologies like satellite-based localisation networks. Their main aim is to manage the handovers and to reduce the delays caused by them in wireless networks. In the world of prediction there are two main approaches:

- the first is based on predicting the prospective movement of the mobile stations by using their previous motions (for example position, velocity) [1] [2] and solving the problem of handovers by appropriate resource allocation 3.

- the supporters of the second theory believe in the methods, based on costumers' conventional behaviour patterns [4] [5. This theory was brought to the front by the Global Positioning System, and most of the applications are about prediction of vehicles' motion.

Chan et al. 2] worked out alternative methods. The first is based on the actual position to predict the next step, reminding us of the first-order Markov Model. The second algorithm is based on actual and former positions, the direction is determined by these data. We work on the generalization of this algorithm. In the third method the routes are segmented and the second algorithm is used on these segments.

Liu et al. 3] explored wireless ATM networks, where they use pattern matching algorithm to predict the next ATM cell during the route. For this reason the examination of cells' rows, measurements of signal strengths and intuitions of the cells' shape is used.

In the early 2000s the development of the mobile phone networks reached the level, when the challenges of networks' management became more complex than prediction of mobile stations' motion. As the result of their evolution the networks became as efficient as the customers and the providers required. The turning-point was that the complex, hypothetical algorithms were surpassed by the simple, algorithms exploitable in practice.

The next technical wave took place with the spreading of the positioning, mainly with the widespread availability of GPS equipments. These technical solutions require more precise prediction ability on one hand, but on the other hand they supplied more usable, precise data. In this area the most promising results were published in the [6] [7. The accuracy of these models were about $70 \%$, but it is necessary to notice that the properties of the environment are inputs for the models. Our aim to define general prediction models, which can be implemented and used easily everywhere, so we require our models to be independent from the platform.

In the literature of the topic generally working prediction models applicable to any environment were not found. We aim to construct universally applicable models, which can collaborate with any tracking system.

\section{Mobility Modelling}

Our study would like to give such a general solution to predict mobility of mobile equipments and their owners that is simple and easy to implement. In our realization the basis of each single method is a graph-model, which can be generated 


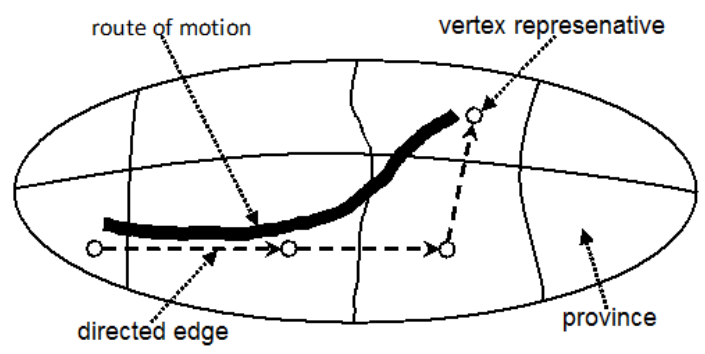

Fig. 1. A given route and its graph representative

by converting the tracking data into graphs. This means that the single directed graph representative of the tracking data is examined, that can be generated from the data according to the required accuracy. The simplest graph creation method is to divide the area into provinces. Let us map the provinces onto the vertices of the graph. We may draw the $a_{i j}$ directed edge of the graph by examining the fixed routes. If we find a route from province $i$ to province $j$, we draw a directed edge from the point $p_{i}$, to the point $p_{j}$. You can see such a directed graph representative in Fig. 1].

The methods described below give alternative solutions to predicting routes in such graph model.

\section{Mobility Prediction Models}

As we have already mentioned, our aim is to define and to analyse such general prediction methods, which can be applied in a wide range, and they can operate in cooperation with the infrastructure of any tracking systems. Probably they are not the most efficient ones in a special environment, but with small-scale transformations they work with any kind of systems. Our elaborated models are classified into 3 groups, such as Simple statistical model, Markov models and Pattern matching models.

\section{Conventional Notations}

Nominate with $p_{i} \cdot p_{j}$ that case, when in a given route at time $t$ our position is the vertex $p_{i}$, at time $t+1$ the vertex $p_{j}$ will be our position. In the following cases we nominate with $p_{i} \rightarrow p_{j}$ those incidences where at a given $t_{1}$ time the position is vertex $p_{i}$, then at a given $t_{2}>t_{1}$ time the position will be vertex $p_{j}$. The models predict the next vertex based on the available information, nominate it with $X$.

The prediction is based on a well-known indicator confidentiality level. We count it with the following formula:

$$
C(X)=\frac{\text { the number of routes crossing series of vertices } P_{X}}{\text { the number of routes crossing series of vertices } P_{i}}
$$


The models can give a probability on a confidentiality level, what the next station of the movement will be.

\subsection{Simple Statistical Model}

One of the tasks attributed to the data mining is the mining of the frequent element sets. The simple statistical model tries to use this to predict routes. The model is very simple, the accuracy of the prediction will be prospectively small. $\left|p_{i}, p_{k}\right|$ nominate the number of the set of routes, contains $p_{i}$ and $p_{k}$ too. At a given vertex $p_{i}$ the model predict $X=p_{k}$ vertex for the next station of the route which satisfy in all cases the following inequatity:

$$
\left|p_{i}, p_{k}\right|>\left|p_{i}, p_{j}\right| \quad \forall j \neq k, \quad j=1,2,3, \ldots
$$

\subsection{Markov Models}

Markov models are originated with Markov chains. We assume that the system and its graph representative are given. In this case the directed graph if there are enough given vertices and edges - behaves similar to the Markov chains. Let us map the vertices of the graph to states of the Markov chain, let us build a matrix from its edges, which can be considered the matrix of transition probabilities. These models are originated with the Markov chain. We may map them to the terminology of the graph model, so Markov models predict the vertex $X=p_{i+1}$ which has the highest confidentiality level in series $\left(p_{i-z} \cdot \ldots \cdot p_{i-2} \cdot p_{i-1} \cdot p_{i}\right) \cdot p_{i+1}$, where the expression in parentheses is known and $z=0 ; 1 ; 2 ; \ldots$ In mathematical phrases: the model predicts that vertex $X=p_{i+1}$, with the highest corresponding $C\left(p_{i+1}\right)$ value. Markov models are not capable of prediction in any situation, when there is vertex, which is not a head of any directed edges or when there are no $z-1$ former vertices of the walk. In practice we implemented and tested the First-, the Second- and the Third-order Markov Models. The depth of the Markov models can be increased, but the demand of memory and storage monotonously rises. By analyzing the former models the following question was formulated in our minds: what kind of results can we get with easing the rigorousness of direct succession or can it help to improve the accuracy of the former models. The pattern matching models were formulated as the result of this intuition. We hope that this will be compensated in increased accuracy and efficiency compared with our former models.

\subsection{Pattern Matching Models}

Pattern matching models are special cases of frequent sequence mining belonging to the category of frequent sample mining. Frequent sequence mining means that we would like to define part series, which are often appearing in given series. The often expression indicates that in the case of the original task we only deal with the series if the number of the existing series among the routes is higher than 
a certain threshold. Unfortunately this algorithm is exponential, the frequent sequence mining demands much time and resources in large datasets. In the case of models drawn up by us, task is not entirely this. On one hand we do not demand a minimal incidence threshold from the series, on the other hand we examine only a certain long incidence of series. We expect longer running time and bigger memory claim from these algorithms, than we have experienced in Markov models. We implemented and tested four Pattern Matching Models. With the Pattern Matching Model No. 1 we ease the constraints of the Firstorder Markov model, instead of $\left(p_{i}\right) \cdot p_{i+1}$, we examine $\left(p_{i}\right) \rightarrow p_{k}$. The Pattern Matching Model No. 2 eases the constraints of the Second-order Markov model, instead of $\left(p_{i-1} \cdot p_{i}\right) \cdot p_{i+1}$, it examines $\left(p_{k} \rightarrow p_{i}\right) \cdot p_{i+1}$. We ease the constraints of the Third-order Markov model with Pattern Matching Model No. 1, instead of $\left(p_{i-2} \cdot p_{i-1} \cdot p_{i}\right) \cdot p_{i+1}$, we examine $\left(p_{k} \rightarrow p_{i-1} \cdot p_{i}\right) \cdot p_{i+1}$. Pattern Matching Model No. 4 is interpreted as the extension of the Pattern Matching Model No. 3 with another former vertex, it means we examine $\left(p_{k 2} \rightarrow p_{k 1} \rightarrow p_{i-1} \cdot p_{i}\right) \cdot p_{i+1}$. The base of the prediction is the highest corresponding $C\left(p_{i+1}\right)$ value.

We can count on the fact that the running time and the memory claim of the pattern matching models will be bigger than the demand of the Markov models. We hope that this will be compensated in increased accuracy and efficiency compared with our former models.

\section{Simulation}

\subsection{Concrete Simulation Environment and Simulation Dataset}

We simulate the tracking in a shopping area like one equipped with RFID (Radio Frequency IDentification) readers and tags. It means we simulate the motion of the customers, and we store their routes in datasets. The datasets contain the identifier of the tag, the identifier of the reader and the time when the action has happened. The simulated model is two-dimensional. The readers are ideal readers, the characteristic of the readers are circles with $10 \sqrt{2}$-radius. The measurements of the simulated area are $90 \times 50$, in which 45 readers watch the motions. The readers are placed in a grid, from the $(5 ; 5)$ to the $(85 ; 45)$ coordinates by 10 units. The customers move in discreet grid in the area simultaneously, one step is one unit.

A public tracking dataset of location-based services was not accessible, therefore a simulation dataset was used to demonstrate the effectiveness of our solutions. Our datasource simulates a customer of a hypermarket, where the topology of shelves, the products and their locations, the product sets bought by the costumers are based on real life. The sufficient quality of the simulated dataset is guaranteed by the complex artificial behaviour of the customer agent, which contains the following aspects:

- The customers enter the hypermarket with an explicit aim represented by a set of products, but they can buy items impulsively as well. 
- When a custromer enters the hypermarket, it has rough map of the topology of the hypermarket and the positions of products, and during its movements this map is improved by new data.

- The agent has a searching strategy to find products with unknown locations, but it has a probability model to give up the searching as well.

The simulation method has an additional RFID tracking layer as well, RFID readers with overlapping effective range track the costumers shopping basket, which contains RFID tags, therefore the precise location of the agents can not be calculated by processing of the dataset of RFID readers. Our input was this simulated tracking dataset to analyse our proposed algorithms.

Divide the area into provinces according to areas seen by $n=1,2,3, \ldots$ readers. In this manner the area is separeted to 121 pieces, that means we get a 121 -vertex graph. The determination of the edges' number is more complicated task, but it is possible if we know the characteristic of the training set. In this situation the number of edges is 888 . The complexity of the graph is evident, nevertheless we try to predict route in the graph.

During generating the simulated data we make the first filtration. We stored the stations of the routes infrequently, only when it changes province, so in the graph terminoligy when it goes across an edge or it steps to another vertex of the graph. We characterize this vertex with the identifiers of the readers seeing it. Since temporal examinations are not made, - this is the reason, why the graph representative can be used - the data is cleaned from the time moments and only the series of the provinces' identifiers are left in the stored data. From this information the routes are unambiguous definable.

After this procedure we get a tracking dataset with 14200 routes, on which the prediction analysis of the models are done. This dataset is divided in two parts: a training set with 12000 and a validation set with 2200 routes. The average length of routes is 126.4 in the training set. We can consider this dataset as big enough to treat the measured accuracy as general.

If the graph is examined an interesting fact can be discovered. The outdegree of the vertices has big variation. It is thanked that, the simulated customers can go in a discrete grid, so they can step into such provinces, which is not neighbouring. The Fig. 2 shows the distribution of the vertices' outdegree. On the horizontal axis of the diagram we represent the outdegree of the vertices, and we depicted the percentile distribution of the vertices on the vertical axis.

\subsection{Results}

Table 1 shows the training time, the validation time, the next vertex's prediction time and the accuracy in percent of the single models. It is visible that the training time of the models are a bit long, but it is offline running time, so it can be accepted, they are needed to run rarely. Intuitive requirement is taken, that the real-time application gives result in 1 second. Our models have to satisfy this statement. 


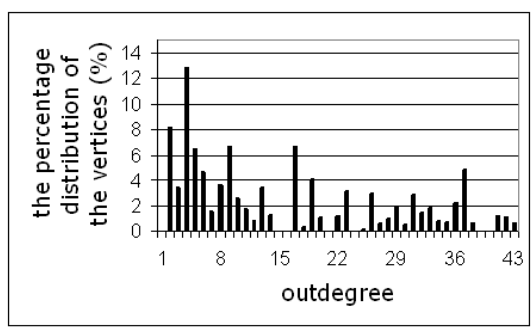

Fig. 2. The distribution of the vertices as the function of their outdegree

Table 1. Comparion of the prediction models

\begin{tabular}{|c||c|c|c|c|}
\hline Model & $\begin{array}{c}\text { Training } \\
\text { Time }\end{array}$ & $\begin{array}{c}\text { Validation } \\
\text { Time }\end{array}$ & $\begin{array}{c}\text { Prediction } \\
\text { Time }\end{array}$ & Accuracy \\
\hline \hline Simple statistical model & $24 \mathrm{~min}$ & $52 \mathrm{~s}$ & $0.18 \mathrm{~ms}$ & $32.4 \%$ \\
\hline First-order Markov model & $27 \mathrm{~min}$ & $51 \mathrm{~s}$ & $0.18 \mathrm{~ms}$ & $49.7 \%$ \\
\hline Second-order Markov model & $3.8 \mathrm{~h}$ & $50 \mathrm{~s}$ & $0.18 \mathrm{~ms}$ & $64.7 \%$ \\
\hline Third-order Markov model & $5.3 \mathrm{~h}$ & $48 \mathrm{~s}$ & $0.17 \mathrm{~ms}$ & $69.2 \%$ \\
\hline Pattern matching model No. 1 & $8 \mathrm{~min}$ & $46 \mathrm{~s}$ & $0.16 \mathrm{~ms}$ & $6.1 \%$ \\
\hline Pattern matching model No. 2 & $6.3 \mathrm{~h}$ & $12 \mathrm{~min}$ & $2.53 \mathrm{~ms}$ & $51.6 \%$ \\
\hline Pattern matching model No. 3 & $12.9 \mathrm{~h}$ & $13 \mathrm{~min}$ & $2.74 \mathrm{~ms}$ & $65.2 \%$ \\
\hline Pattern matching model No. 4 & $17.8 \mathrm{~h}$ & $12 \mathrm{~min}$ & $2.51 \mathrm{~ms}$ & $51.9 \%$ \\
\hline
\end{tabular}

The simulations's results of the Markov models are seen in the Fig. $3(\mathrm{~b}), 3(\mathrm{~d})$ It is observable that the distribution of the models are almost the same, the diagrams are similar to each other, there are at the same place under and above the average. The variation of the distributions are almost the same, there are deviations only between the average values by the difference in elevations. The results meet our expectations, because there origin is the same first-order Markov Model. The accuracies of the models are 50, 65 and $69 \%$. It is noticeable that completed with newer former vertices the accuracy is rising, but the degree of the improvement is falling. It means the marginal utility of a newer vertex is falling. The improvement of plus one vertex is not rewarding, because the demand of the memory and the storage is rising more.

The accuracy distributions of the pattern matching models (Fig. $3(\mathrm{f})+3(\mathrm{~h})$ except for the Pattern matching model No. 1 - are very similar to each other, the variations of them are almost the same, the difference is only the average value. We can parallel them to the Markov models, the distribution of the accuracy is similar. It was expectable, because the origin of the models is the first-order Markov model. It is seen that the unstretching of the rigorousness of the direct following make the model less accurate, but on the other hand the added former vertex can improve the efficiency of the models. Nevertheless the training time 


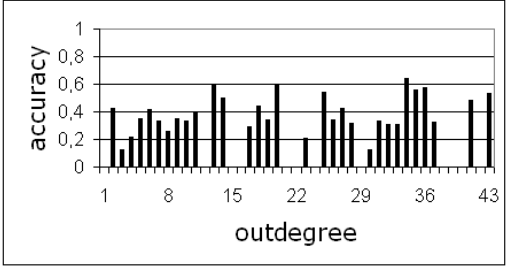

(a) Simple statistical model

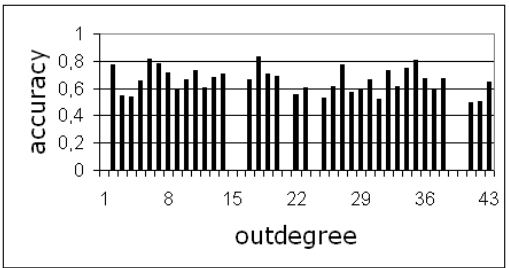

(c) Second-order Markov model

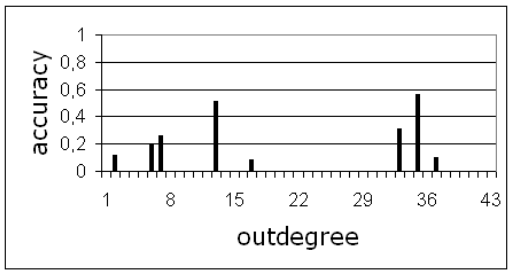

(e) Pattern matching model No. 1

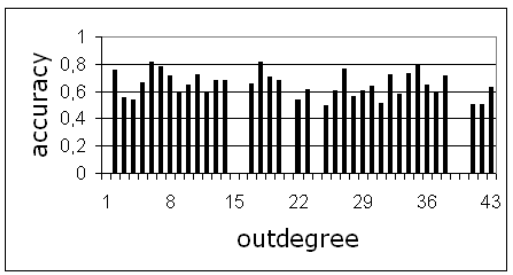

(g) Pattern matching model No. 3

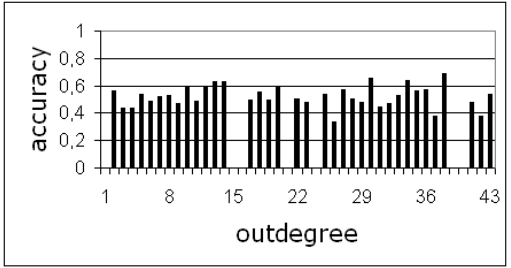

(b) First-order Markov model

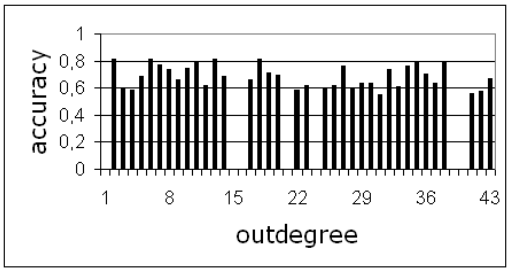

(d) Third-order Markov model

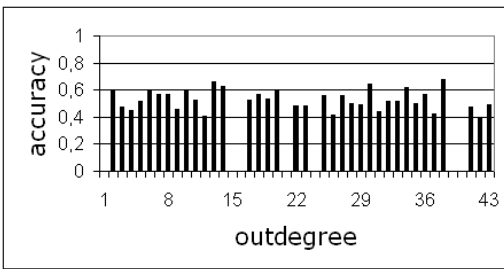

(f) Pattern matching model No. 2

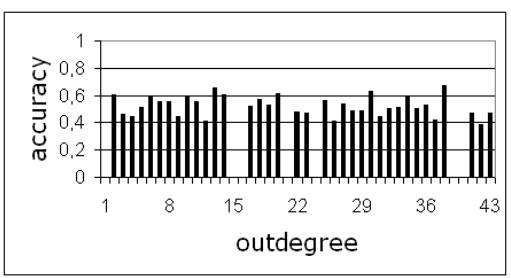

(h) Pattern matching model No. 4

Fig. 3. The accuracy of the models as a function of the outdegree of the vertices

increases, and it is not worth this increase, because nearly the same accuracy can be reached with simpler models.

Analysing the Pattern matching model No. 1 (Fig. 3(e) is very interesting, because it is very different from the others. We expect more efficiency from this model based on the success of the simple statistical model, because in that case we add the vertices, which were visited in the past. We can get the conclusion from that the examined area is closed. There are some outgoing vertices, and the customers leave the shopping area once. The pattern matching model No. 1 predict these vertices all the time. This method can predict right vertex, only if the customer is at the last but one station of his route. 
By analysing the figures above we can notice another interesting fact, the accuracy of the prediction depends on the outdegree of the vertices only in small range, meaning it does not depend on the number of the potential next vertices. We can deduce that there are some typical routes in the shopping area, and there are only some individuals, who deviate from this. These individuals increase the outdegree of the vertices, but they can not affect the typical routes. In a future improvement of the model, we only add an edge to the graph, if there are more routes, across it, than a given threshold.

\section{Conclusion}

The success of the location-based services can be improved with mobility prediction. We gave an alternative solution for this. We defined 4 types of models, which are based on the representative graph, that can be built up by the area separated into provinces. Despite of the intuition that the simple statistical model can not be used to predict the next station of the routes, it can successfully predict that in $30 \%$ of the cases. The Markov models are originated with the Markov chains. The models use only the current and some direct former vertices for prediction. If we use these models we can reach about $70 \%$ accuracy. Many models were published in which the accuracy is about $60 \%$, still they are used. The pattern matching models are to improve the efficiency of the Markov models. This approach can help a bit, but the offline running time increase more.

The actual published models can be improved, because the accuracy can be increased by training at specific environment. The effects the environment can be trained by the models, and it makes them more useable in the marketing world, or to in finding acquaintances in the users' neighbourhood.

\section{References}

1. Junius, M., Kennemann, O.: New Methods for Processing GSM Radio Measurement Data: Applications for locating, Handover, and Network Management. In: Vehicular Technology Conference, vol. 44(1), pp. 338-342 (1994)

2. Chan, J., et al.: A QoS Adaptive Mobility Prediction Scheme for Wireless Networks. In: Global Telecommunications Conference, vol. 3, pp. 1414-1419 (1998)

3. Liu, T., Bahl, P., Chlamtac, I.: An Optimal Self-Learning Estimator for Predicting Inter-Cell User Trajectory Wireless Radio Networks. In: Proceedings of the IEEE International Conference on Universal Personal Communications, San Diego, California, USA, pp. 438-442 (1997)

4. Schonfelder, S.: Some Notes on Space, Location and Travel Behaviour. In: 1. Swiss Transport Reseach Conference, Monte Verita, Ascona (2001)

5. Gartner, N., Messer, C.J., Rathi, A.K.: Traffic Flow Theory. A state-of-the-Art Report, http://www.tfhrc.gov/its/tft/tft.htm

6. Liao, L., Patterson, D.J., Fox, D., Kautz, H.: Building Personal Maps from GPS Data. Annals of the New York Academy of Sciences 1093, 249-265 (2006)

7. Krumm, J., Horvitz, E.: Predestination: Inferring destinations from partial trajectories. In: Dourish, P., Friday, A. (eds.) UbiComp 2006. LNCS, vol. 4206, pp. 243-260. Springer, Heidelberg (2006) 